\title{
Quality Assurance of Grain with Colour Line Scan Cameras
}

\author{
Brückner, Peter; Anding, Katharina; Weißensee, Holger; Dambon, Martin \\ Technische Universität IImenau, Quality Assurance Department \\ P.O. Box 100 565; 98684 IImenau (Germany)
}

\section{INTRODUCTION}

Grain especially wheat is one of our most important foodstuffs. A lot of strict regulations exist for the quality assurance of grain. The strict quality criteria contain prescriptive limits for several fractions in grain, especially for toxic and unsound constituents as well as specific baking properties of flour. There are already many procedures and equipment for the determination of specific baking properties like gluten content, protein content, falling number and moisture content of grain. But the acceptance of cereal in mills is still based on a subjective visual inspection by a miller or a laboratory assistant. This kind of quality control is time consuming and error-prone. The manual analysis of elements in a grain sample is called "Besatz analysis of cereal" and is described in the ICC standard methods from the International Association for Cereal Science and Technology [1], [2]. The main fact of this method is to separate all groups of Besatz from the normal basic grains by sieving and manual selection. Besatz are all matters of a sample of grain which are other than the basic cereal of unimpaired quality. The unhealthy and noxious impurities (a part of the so called miscellaneous impurities), e.g. fungus-covered grain (in particular with fusarium) and ergot, have a specific relevance because of the negative effects of the metabolic waste products of fungi on humans and animals.

The existence of noxious substances like fusarium toxins (e.g. Deoxynivalenol, Zearalenon) can be detected by using rapid diagnostic tests without accurate results or by time-consuming laboratory tests like immune-chemical methods (ELISA) or chromatographic methods (thin layer chromatography, high performance liquid chromatography) with high-precision results.

But often a grain delivery requires a very fast decision for acceptance or refusal with regard to compliance with quality criteria.

So the results of the Besatz analysis of cereal are very deciding for acceptance or refusal of the delivery by lorry, railway-car or ship and therefor in case of acceptance for adequate payment.

In this context conventional methods like the manual Besatz analysis are disadvantageous because of their high subjective and time-consuming characteristics and their small sample sizes with low confidence level.

\section{RESEARCH}

An automated object recognition routine for the Besatz analysis of grain is the task to be solved. The aim of an automated digital imaging analysis of grain quality should be achieved with efficient, objective and fast optical analysis. So the aims of an automatic Besatz analysis are objective decisions, higher confidence level by using larger sample sizes and reduced time for decisions.

The comparison of manual and automatical analysis of Besatz is shown in Table 1.

\begin{tabular}{|c|c|c|}
\hline & $\begin{array}{l}\text { manual analysis of Besatz } \\
\text { (impurities) }\end{array}$ & automatic analysis of Besatz \\
\hline sample size & $50 g-100 g$ & $250 g-500 g$ \\
\hline time of analysis & $30 \mathrm{~min}-45 \mathrm{~min}$ & $5 \min -10 \min$ \\
\hline evaluation & subjective & objective \\
\hline
\end{tabular}

Table 1: Comparison of manual and automatic analysis of Besatz

The basic procedure of the automatic analysis of Besatz is shown in Figure 1. 


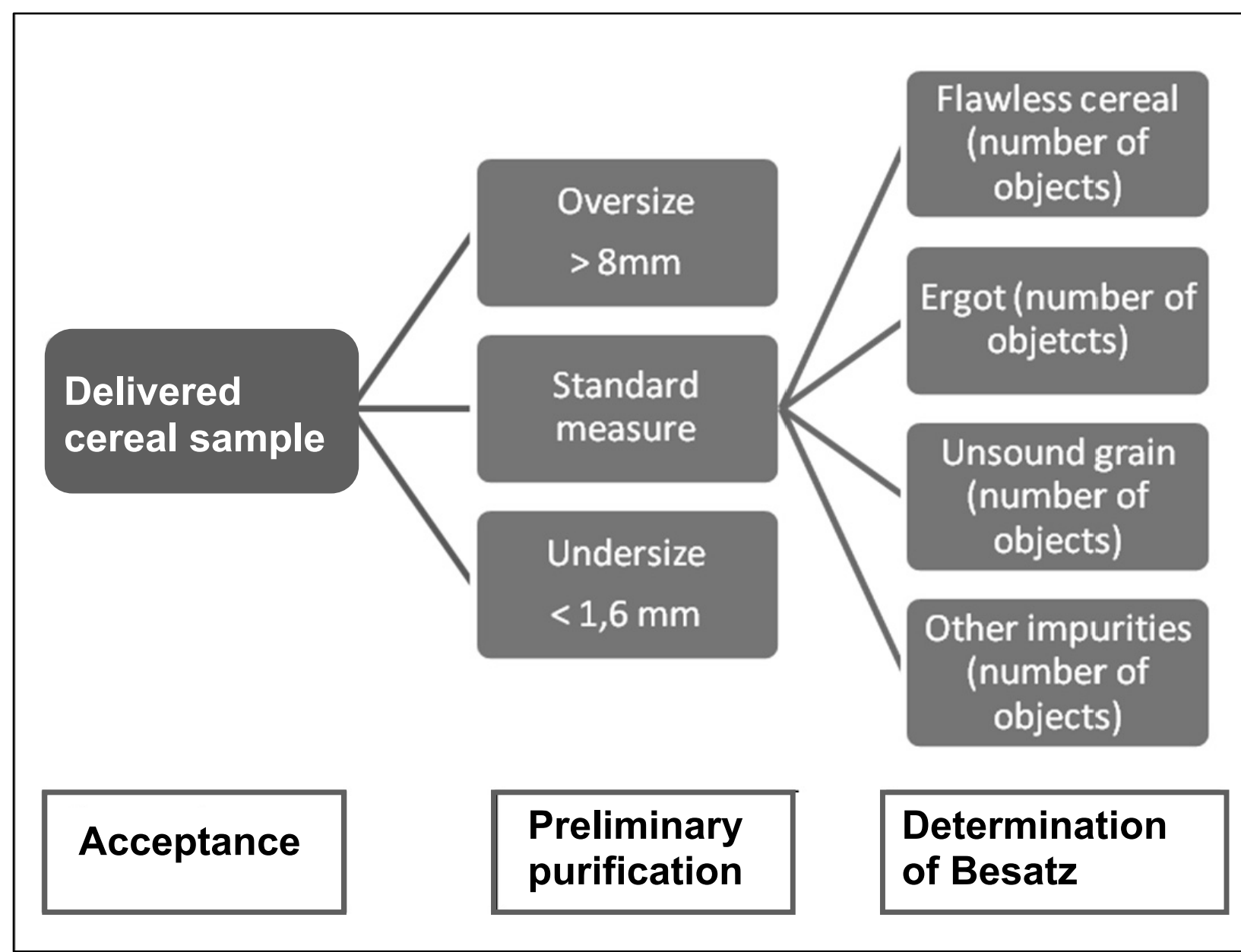

Figure 1: Basic procedure of the automatic analysis of Besatz

The three steps of automatic analysis are acceptance, preliminary purification and determination of Besatz. The primary results of the automatic analysis are the number of objects of the four main classes: flawless cereal, Ergot, unsound and bunted grain and other impurities.

In legal requirements for Germany and European Union the different fractions in the grain sample have to be denoted in percent by weight related to the total weight of the sample. This requires a weighing of the total sample before analysis and a weighing of the different fractions particularly the fraction flawless cereal after analysis. This means that besides the electronic, easily practicable counting of all objects from each class a automatic grading have to be occured. Such an automatic grading requires fast decision times for data processing.

The technical basic principle for the automatic analysis of Besatz is based on the image acquisition with a RGB-CCD line scan camera (A) and object feeding in free fall (see Figure 2). An illumination system consisting of front and transmitted light units is practical to illuminate the cereal sample. Optional it is possible to install a second CCD line scan camera (B) with its own illumination system. Another important additional option is a pneumatic sorting system after image acquisition and recognition routine. In particular, according to the regulations the different fractions have to be evaluated in percent by weight. If the fractions are separated after classification it will be very straightforward to evaluate the fractions in percent by weight. But this evaluation can also be based on a mass calculation of the cereal sample using the re-corded area or generate the volume of the analysed objects. In this case the mass is calculated by the specific density and generated volume of the object. Variation in density and generated volume can be compensated to a certain degree by averaging over a high number of objects of the specific class.

In image processing applications the problem solution is fundamentally based on the alternative between line sensor systems and matrix sensor systems. Furthermore in this case a decision has to be made whether to inspect the objects on a conveyor belt or in free fall. The comparison of the pros and cons of the four variants shows, concerning to the analysis of Besatz, that the solution with line scan cameras and image acquisition in free fall forms many advantages (see Table 2). 


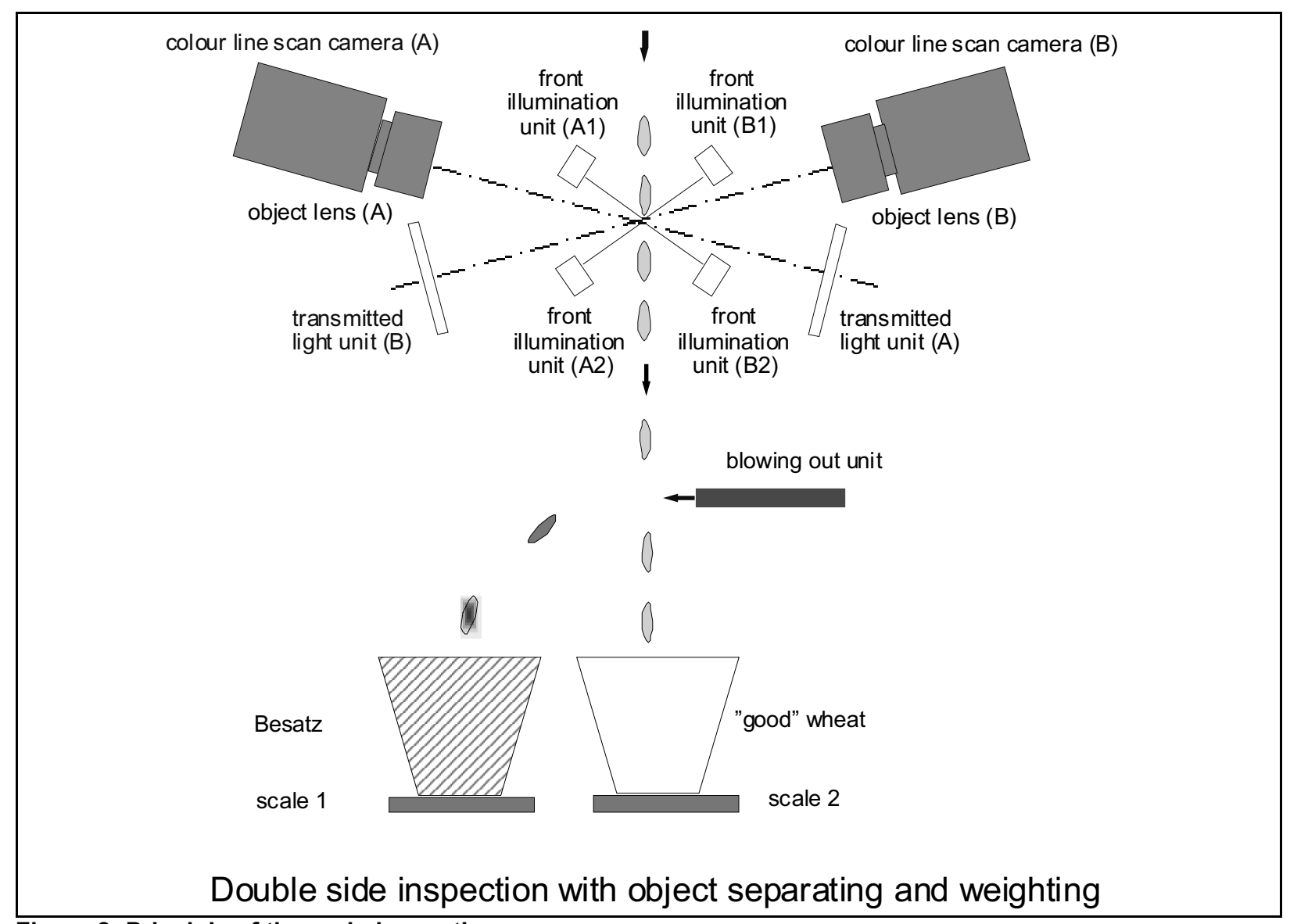

Figure 2: Principle of the grain inspection

\begin{tabular}{|c|c|c|c|c|}
\hline \multirow[t]{2}{*}{ criterion } & \multicolumn{2}{|c|}{ use of line scan camera } & \multicolumn{2}{|l|}{ use of matrix camera } \\
\hline & conveyor belt & free fall & conveyor belt & free fall \\
\hline soiling tendency & yes & no & yes & no \\
\hline acquisition speed & low level & high level & low level & high level \\
\hline object field & 1 dimensions & 1 dimensions & 2 dimensions & 2 dimensions \\
\hline illumination options & limited & flexible & limited (flash light, shutter) & flash light \\
\hline illumination control & no & no & yes & yes \\
\hline $100 \%$ inspection & $\begin{array}{l}\text { yes (with feed rate } \\
\text { signal) }\end{array}$ & $\begin{array}{l}\text { yes (with trigger } \\
\text { signal) }\end{array}$ & $\begin{array}{l}\text { limited (only with feed rate } \\
\text { signal) }\end{array}$ & no \\
\hline double side inspection & no & yes & no & difficult \\
\hline $\begin{array}{l}\text { effect of object motion } \\
\text { (rotation) }\end{array}$ & present & present & little & little \\
\hline automatic sorting & yes & yes & yes & difficult \\
\hline \multirow[t]{2}{*}{ cost comparison } & \multicolumn{2}{|c|}{$\begin{array}{l}12 * 10^{6} \text { pixel / triple-line RGB-CCD } \\
\text { - price ca. } 5000 \text { Euro }\end{array}$} & \multicolumn{2}{|c|}{$\begin{array}{l}16 * 10^{6} \text { pixel / one-chip RGB-CCD } \\
\text { - price ca. } 15000 \text { Euro }\end{array}$} \\
\hline & & & $\begin{array}{l}15 * 10^{6} \text { pixel / triple-chip } R C \\
\text { - price ca. } 35000 \text { US-Dollar }\end{array}$ & B-CCD \\
\hline
\end{tabular}

\section{Table 2: Comparison of different hardware variants}

The free fall is an accelerated motion with constant acceleration. In small areas, like the object field of a line scan camera, the accelerated motion can be approximated as a linear motion with constant velocity. Therefor the exact object velocity is necessary for the image acquisition with a line scan camera.

A simple and precise method to solve this problem is to record a test image of a sphere gauge in the object field of the line scan camera (see Figure 3 ). This sphere shows only a circular outline in the recording if the line scan frequency is properly adjusted. After this calibration the image acquisition does not have any distortions. 


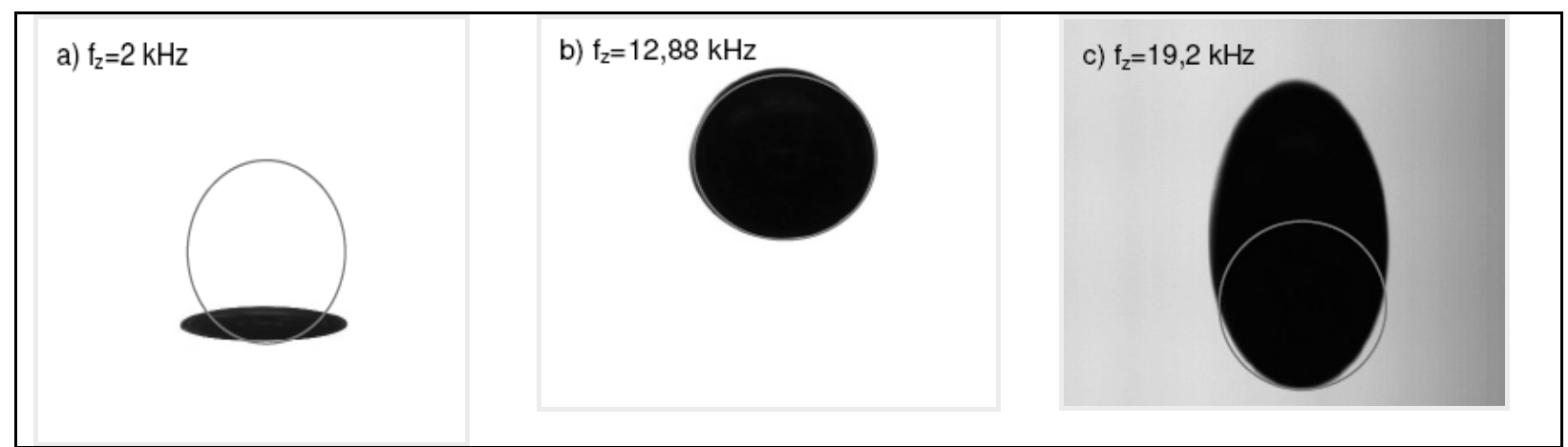

Figure 3: Finding the optimal line scan frequency

$$
n(\text { Pixel })=\frac{B}{D_{x}} \quad v_{O}=\sqrt{2 \times h[\mathrm{~mm}] \times 9810\left[\mathrm{~mm} / \mathrm{s}^{2}\right]} \quad f_{\text {hor }}=\frac{v_{O}}{D_{x}}
$$

The optimal line frequency subject to number of pixels, resolution and falling speed is shown in Table 3 .

\begin{tabular}{|l|l|l|l|l|l|l|}
\hline $\begin{array}{l}\text { number of } \\
\text { pixels }\end{array}$ & object field & $\begin{array}{l}\text { resolution } \\
\text { (object field) }\end{array}$ & falling height & falling speed & $\begin{array}{l}\text { line } \\
\text { frequency }\end{array}$ & pixel clock \\
\hline $\mathrm{n}$ & $\mathrm{Dx}$ & $\mathrm{b}$ & $\mathrm{h}$ & $\mathrm{v}_{0}$ & f line & $\mathrm{f}_{\text {clock }}$ \\
\hline & {$[\mathrm{mm}]$} & {$[\mathrm{mm}]$} & {$[\mathrm{mm}]$} & {$[\mathrm{mm} / \mathrm{s}]$} & {$[$ lines $/ \mathrm{s}]$} & {$[$ pixels $/ \mathrm{s}]$} \\
\hline \multirow{2}{*}{2048} & \multirow{2}{*}{204,8} & 0,1 & 50 & 990 & $10,1 \mathrm{kHz}$ & $20,8 \mathrm{MHz}$ \\
\cline { 4 - 7 } & & & 100 & 1401 & $14,3 \mathrm{kHz}$ & $29,4 \mathrm{MHz}$ \\
\hline
\end{tabular}

Table 3: Overview of dependency between pixel number, falling speed and chosen line frequency

There are three fundamental technical approaches: the one-line scan CCD with RGB-Bayer-pattern colour filter array [3], the triple-colour scanning lines where the colour lines (red, green, blue) are placed one below the other and triple-colour line scan camera with beam splitter prism that the RGB scanning lines are in the same axis of vision. The second type of camera with three scanning lines was extended by a fourth line to enlarge the spectral sensitivity for the near-infrared (NIR). Another solution statement for the NIR-sensitivity is a variation of the triple-colour-line-principle. The function of this variation is to combine the blue and the green array in the first and the red array in the second channel. In this way it is possible to use the third channel for the NIR.

The expansion of the visible electromagnetic spectrum to the near-infrared is reasonable because of the technology of the installed CCD chips already include the sensitivity up to $1100 \mathrm{~nm}$. Furthermore there are some technical and mainly biological characteristics, which especially take shape in the NIRspectrum. With the expansion of the electromagnetic spectrum it is necessary to modify the illumination units and the object lenses.

Sometimes it is desirable to use the entire NIR spectrum up to $2500 \mathrm{~nm}$, but the built in Si-based sensors in this line scan cameras are limited up to 1100. In this case it is required to use sensors which are based on the InGaAs technology. However this kind of sensors does not detect the visible spectrum.

An automated recognition routine for analysis of grain quality was developed by the authors, especially the recognition and classification of normal basic grains and fractions of Besatz was investigated. Each fraction generates an object class (such as wheat, rye, stones,..). The automated analysis accomplishes a time saving of about $80 \%$ compared to the manual analysis, using identical or even greater samples quantities. The confidence level increases significantly with greater sample quantities.

The recognition of the individual objects occurs in a multilevel process (see Figure 4). At first the relevant image information was selected out of the volume flow from the camera by a special method which was developed. Here it is very important that the cut objects are complete. This data reduction on relevant image information reduces the data flow radical by 80 to $90 \%$.

The relevant image information the "single kernel images", are extracted for the following recognition routine (see Figure 5). 


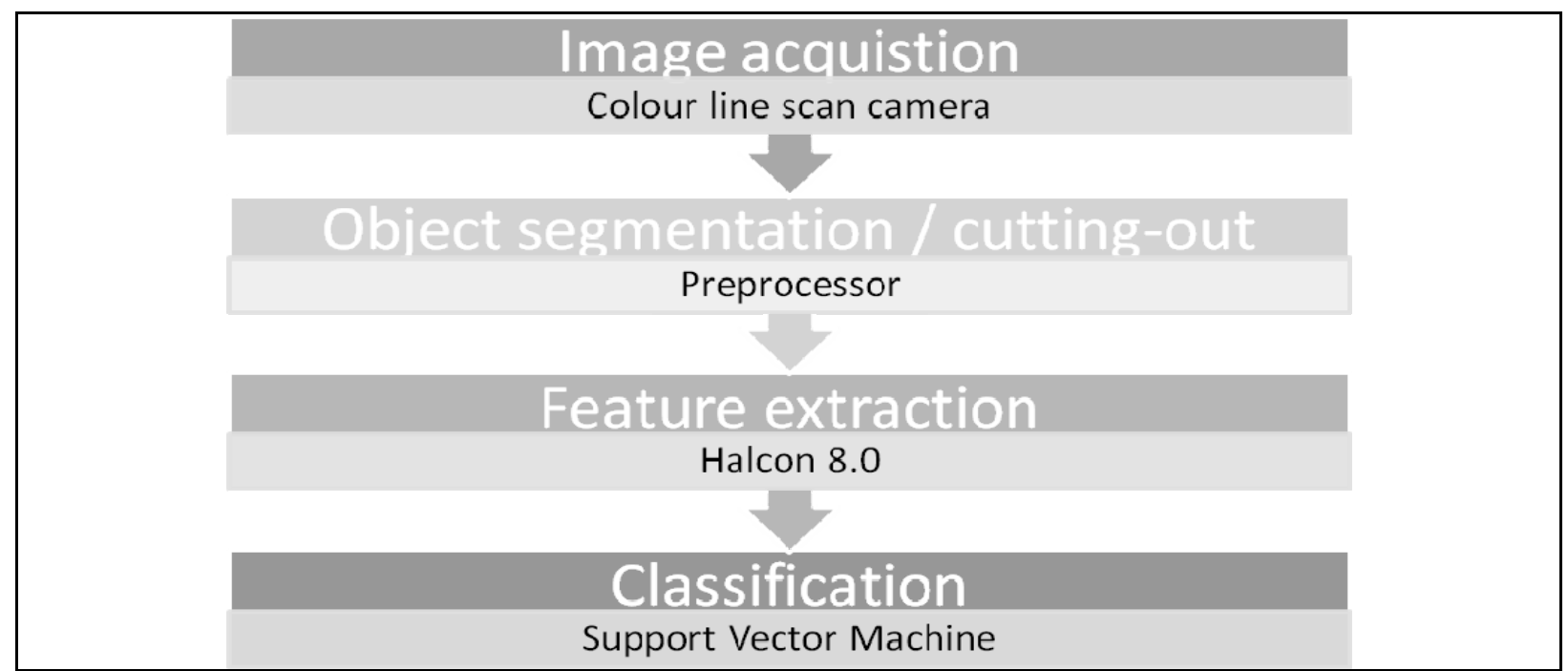

\section{Figure 4: Main steps of the classification process}

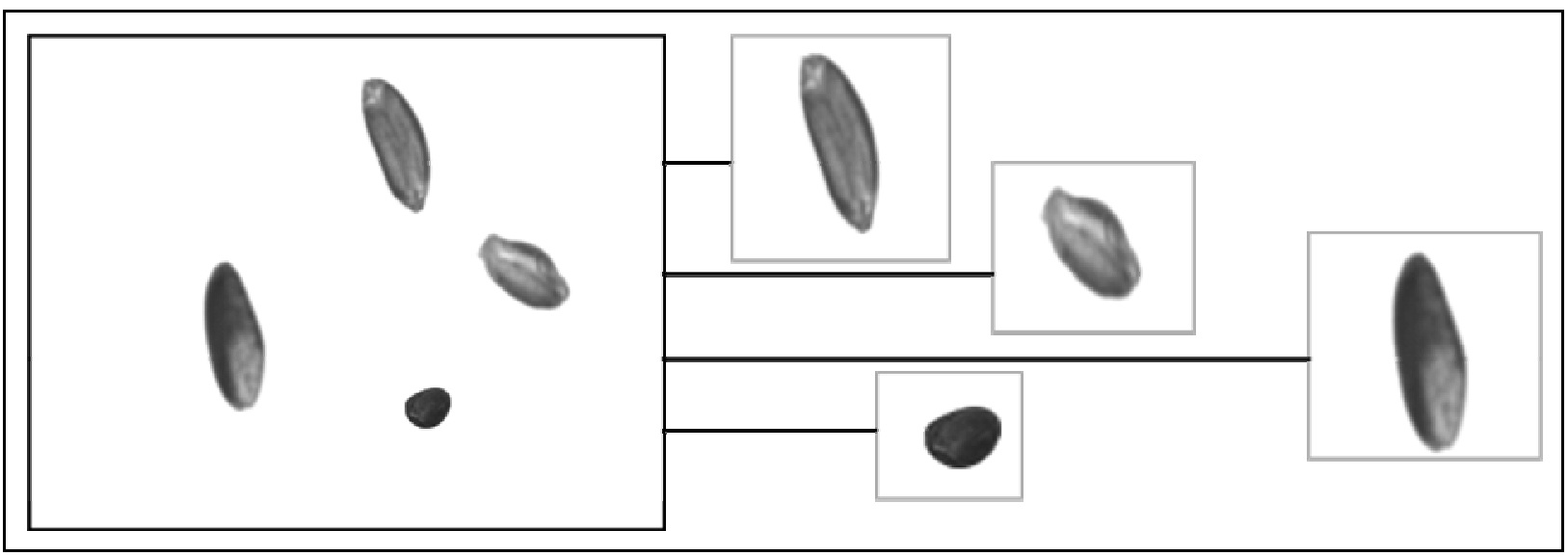

Figure 5: "Single kernel images" - the extracted relevant image information

An adequate number of objects in training data set for each class is a necessary precondition for a successful training process. The required training and test data set is composed of harvest from the last years by Max Rubner-Institut (Federal Research Institute of Nutrition and Food) in cooperation with several german mills. So a database for wheat could be constructed. All class samples should reflect the characteristic variety of the class.

Many object classes from different cereal varieties (wheat, rye, durum, and oat), broken grains of wheat, different grain impurities of wheat, sprouted grains of wheat and different miscellaneous impurities were used in this work. Samples of the 14 object classes flawless wheat, broken wheat, wheat damaged by pests, sprouted wheat, shrivelled wheat, durum, oat, rape, rye, sunflower seeds, weed seeds, husks, stones and contaminations are pre-classified by a human expert (see Figure 6).

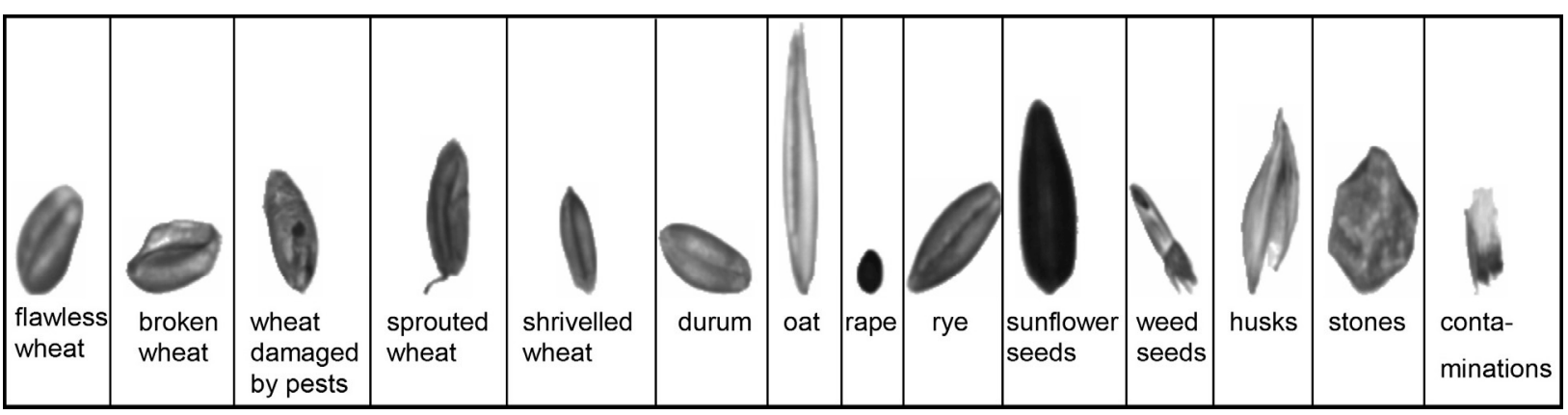

Figure 6: Dataset samples for different object classes 
During the recognition routine all properties of the objects (colour, shape and texture features) are calculated and evaluated. This step is so called feature extraction. The combination of different feature values builds the feature vector which consists of about 188 feature values.

Different classification algorithms were tested on this complex recognition problem to determine their generalization ability. We mainly used the machine learning toolbox "Weka 3.5" and the commercial machine vision library "Halcon 8.0".

\section{OUTCOMES}

Our approaches demonstrated Support Vector Machines (SVM) as the best classification algorithms for this recognition problem. The best SVM with recursive feature elimination (RFE) [4] implemented in Weka [5], achieved a total recognition rate of about $90 \%$ for this dataset and individual recognition rates range from $78 \%$ to $99 \%$ for the different classes (see Figure 7 ).

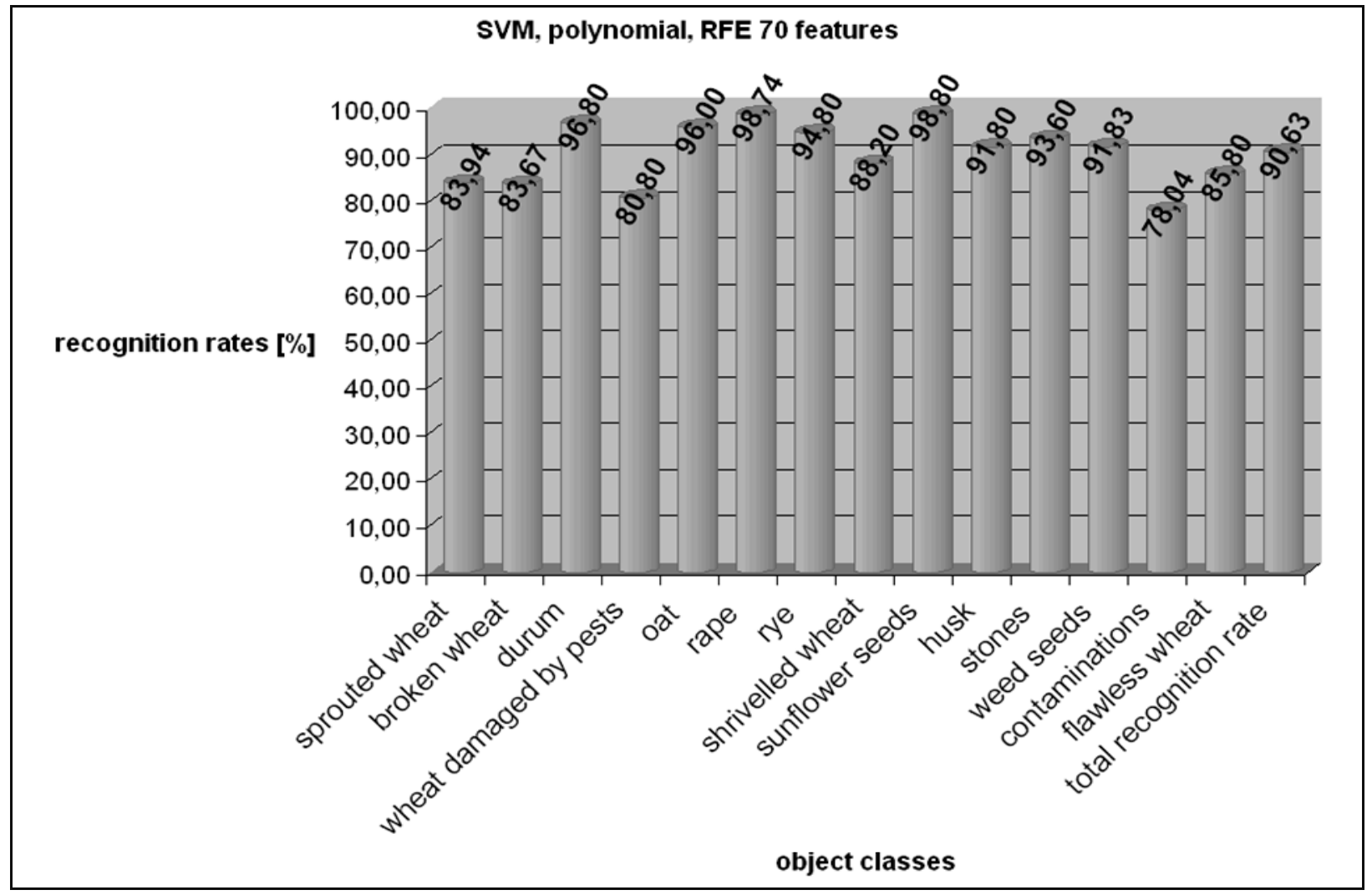

Figure 7: Recognition rates of the 14 object classes from the best tested SVM

The results especially the recognition rates shown, that recognition of classes from natural products is an extensive and sophisticated challenge. In future challenges we have to find new adapted feature algorithms, optimal feature selection methods and optimize the dataset.

\section{ACKNOWLEDGEMENTS}

A project funded by the Federal Ministry for Economic Affairs and Technology under the promotional reference $16 \mathrm{INO} 496$ forms the basis of this paper. The responsibility for the content of this paper lies with the author.

\section{BIBLIOGRAPHY}

[1] ICC No. 102/1: Determination of Besatz of Wheat. International Association for Cereal Science and Technology, 1972

[2] ICC No. 103/1: Determination of Besatz of Rye. International Association for Cereal Science and Technology, 1972

[3] B. E. Bayer: Color imaging array, U.S. Patent 3971 065, 1976

[4] I. Guyon et al.: Feature Extraction, Foundations and Applications. Physica-Verlag, Springer, 2006

[5] I. Witten, E. Frank: Data Mining: Practical Machine Learning Tools and Techniques. Morgan Kaufmann Publishers, 2000 